\title{
HIPPOCAMPAL VOLUME AND MEMORY DEFICITS WITH PERINATAL STROKE
}

Investigators from University of California and Rody Children's Hospital, San Diego, CA, measured the volume of the hippocampus in 27 children with perinatal stroke and 19 controls, and measured their performance on standardized verbal and non-verbal memory tests. As a group, children with perinatal stroke had smaller left and right hippocampi compared with normal controls. Individually, children with perinatal stroke had either 1) no hippocampal loss, 2) unilateral hippocampal loss, or 3) bilateral hippocampal loss. Hippocampal volume is inversely correlated with memory test performance in the perinatal stroke group; smaller left and right hippocampi related respectively to poorer verbal and non-verbal memory test performance. Patients with seizures were more likely to have smaller hippocampal volume than controls and a significantly lower IQ than patients without seizures. Patients with seizures were impaired on 4 of 6 measures of the Children's Memory Scales. (Gold JJ, Trauner DA. Hippocampal volume and memory performance in children with perinatal stroke. Pediatr Neurol 2014 Jan;50(1):18-25).

COMMENTARY. In addition to the loss of hippocampal volume and impairment in memory tests, this study demonstrates that the left and right hippocampi preferentially support verbal and nonverbal memory, and the occurrence of seizures after perinatal stroke may limit the natural postinjury plasticity of the immature brain. The continued treatment of seizures after initial control in infants is a controversial subject. This study suggests that aggressive seizure control beyond the perinatal period may improve cognitive and memory outcomes in children who suffer perinatal stroke.

Treatment of clinical and subclinical seizures after perinatal stroke. The incidence of postneonatal epilepsy and neurodevelopmental outcome in 206 term infants treated with AEDs for neonatal seizure discharges detected by amplitude-integrated EEG (aEEG) in addition to clinical seizures was assessed at 3, 9, 18 months and 3 and 5 years of age. Overall mortality was $39 \%$, and 41 of 126 survivors $(33 \%)$ were abnormal at follow-up; 12 developed postneonatal epilepsy (9.4\%). In 84 children who survived after hypoxic-ischemic encephalopathy grade II, 6 (7\%) developed postneonatal epilepsy; no postneonatal epilepsy was observed if seizure were controlled within 48 hours after birth and when not more than 2 AEDs were required. In 11 children who survived after perinatal arterial stroke, $2(18 \%)$ developed postneonatal epilepsy. The incidence of postneonatal epilepsy after treatment of both clinical and subclinical neonatal seizures detected with continuous aEEG was $9.4 \%$. This was lower that that previously reported in children who received AED treatment for clinical seizures only [1].

\section{References.}

1. Toet MC, et al. Pediatr Neurol. 2005 Apr;32(4):241-7. 